dental research. Three distinct themes, dental hard tissues, oral microbiology, and saliva, are covered. Four chapters relate to the molecular biology of the dental hard tissues. These include genetic control of the dentition, enamel proteins, and immunolocalisation strategies, enamel protein genes and their expression, and, lastly, dental mesenchyme and the expression of molecules therein. One chapter is devoted to the molecular genetics of oral bacteria and the microbial aetiology of dental caries and periodontal diseases. The remaining section deals with salivary composition, with separate chapters on salivary mucins, salivary proteins, and the proline rich salivary proteins. The chapters are contributed by distinguished researchers in each field. Some chapters appear somewhat subjective but this reflects the relative contribution of the author(s) to their subject of interest. The comprehensive list of references at the end of each chapter will provide a valuable source of further reading for those seeking to further their knowledge of the field. Although the editor acknowledges in the foreword that the content of the book will soon become dated because of the rapid advances in molecular biology both in general and in relation to dental research, this book is to be welcomed for bringing together in one volume current thinking regarding molecular biology as applied to the study of oral and dental structures and tissues.

M J ALDRED

Advanced Techniques in Chromosome Research. Ed Kenneth W Adolph. (Pp 462 $£ 166 \cdot 75$.$) New York: Marcel Dekker. 1991$.

Over the last 20 years the purely descriptive field of cytogenetics, and the reductionist, analytical field of molecular biology have steadily approached each other until it is almost time for them to celebrate their wedding. Although it is not yet possible to construct a detailed model of chromosome organisation, the ways in which genes are organised in chromosomes and chromosomes in nuclei are beginning to be well understood, so that the present volume makes an appropriate appearance. However, the value of this book as a contribution to this important field is more debatable.

The title leads one to expect a series of detailed descriptions and evaluations of techniques at the leading edge of chromosome research, whereas what we find is a mixture of review articles (some quite excellent) and technical chapters, with the majority of the latter lacking sufficient detail to make them an adequate guide to implementing the techniques described. There is also a problem (presumably arising from the delay between submission of the manuscripts and publication) with material becoming outdated. Very few of the references cited by most of the authors are later than 1989, and in such a rapidly changing field, this is usually too long ago!

In a volume of such diverse character, I feel that the best way to give an idea of the value of the book is to run briefly through the chapters, with a brief description and comment on each. The book is divided into three sections, covering Molecular Techniques, Cytogenetic and Linkage Analysis, and Cellular Techniques respectively.

The first section begins with a review of 'Molecular diagnostics' (Summar and Phillips), which is detailed and well written, my only criticism being that mentioned above, that there is no reference more recent than early 1989 . This is followed by a chapter on 'Cloning and analysis of large DNA molecules' (Scherer and Tsui). This is primarily concerned with YAC technology and the analysis of YAC DNA by pulsed field gel electrophoresis (PFGE). There is thorough coverage of this important area, but, although protocols are given, they tend to lack the precise details necessary to translate them into successful laboratory procedures. The next chapter is a detailed report of a single piece of work involving the 'Molecular analysis of a single chromosome sub-band' (Yunis). While this is an excellent paradigm for the sort of work involved in molecular cytogenetics, it is not sufficiently universal for the reader to be able to apply it to similar problems in a different system. Chapter 4 describes a method for purifying DNA fragments by immobilised oligonucleotide probes as a stage in high efficiency cloning (Wada, Tsurui, and Suyama). Although the data are presented in great detail, again I feel that the protocols are not sufficiently detailed to be useful as laboratory guides without further amplification. Chapter 5 covers 'In situ hybridization of oligonucleotide probes' (Nanda, Schmid, and Epplen). This is an excellent chapter, reporting a new approach in good and adequate detail. Chapter 6, on the 'Analysis of flow-sorted chromosomes' (Shimizu and Minoshima) has useful protocols, but limited application owing to the use of a single fluorochrome and laser, with the limited resolution available with this system. Again, the most recent references are 1989. The last chapter in this section covers 'Pulsed-field gel electrophoresis', and I am grateful to John Maule for reading it and providing me with his comments. Although it provides a basic introduction to $P F G E$, the experimental procedures are not sufficiently detailed or accurate to allow a novice to carry them out. Some of the figures are of poor quality and the legends are, in some cases, inaccurate, while some of the information is out of date, and some of the cited references are inappropriate. Although the list of references is extensive, there is again nothing after 1989.

The second section begins with a chapter on the 'Analysis of chromosomal alterations in human solid tumors' (Meese and Trent). This is a useful review, though it omits to point out that classical analysis of complex translocations by banding is subject to error, and chromosome painting by fluorescent in situ hybridisation (FISH) often shows very different conclusions and detects translocations overlooked by classical analysis. Chapter 9 is a comprehensive review of the whole subject of 'Fragile sites on human chromosomes' by Sutherland, the doyen of the field, while chapter 10 is again a review, in this case of 'Immunocytogenetics', again by two of the leading exponents of the field, Haaf and Schmid. It is primarily a historical survey of the development of the subject, from the early 1970 s up to 1989 . The remaining three chapters in this section cover aspects of gene linkage studies. As D'Eustachio, the author of the first of these ('Molecular linkage genetics of the mouse') says, construction of linkage maps is not a new activity, and it is difficult to see what these chapters, however sound they may be (and I certainly have no criticism of this one), are doing in a book with the title this one bears. The second of the linkage chapters discusses 'Genome duplication and compar- ative gene mapping' (Nadeau), an interesting and important subject. However, the data are so poorly presented, either because of careless proof reading or errors in composition, that the value is much reduced. The final chapter in this section, 'Human linkage analysis: strategies for locus ordering' (Weeks) presents an advanced mathematical and statistical approach to the topic, likely to be of value to dedicated constructors of maps.

The final section, on 'Cellular techniques', begins with the editor's own contribution, on the 'Arrangement of chromosomes in human mitotic cells'. This describes the construction of 3D images by computer graphics from electron micrographs of serial section through mitotic cells. This seems to me an interesting piece of work, though less valuable than the work by Heslop-Harrison et at (1988) cited by the author, which applies a similar approach to analysing the organisation of individual chromosomes in cells. This is followed by a chapter entitled 'Production of transgenic animals' (Gordon). The first part of this chapter is a thorough review of this topic, but the remainder covers a series of subjects more akin to subfertility studies than basic science. These include micromanipulation fertilisation, pronucleus removal, and prenatal diagnosis by embryo biopsy. The next chapter is a review of 'Mutagen testing using mammalian cells' (Hsu and Elder) and is again a useful review without being in any way a technical guide. The penultimate chapter may be considered as an appendix to that by Meese and Trent in the second section, as it describes the 'Culture of solid lung tumors for cytogenetic analysis' (Siegfried et al). Again, while technical information is given, it is not in sufficient detail to permit a novice to perform the procedures. The last chapter discusses the use of 'Colchicine and colcemid to block microtubule assembly' (Sluder) and, again, while it is a first class research report, the methodology is not described in sufficient detail for it to make an adequate laboratory guide.

In summary, this volume may have a misleading title, as the majority of the contributors do not provide more than a review of the field covered by their titles, and few of those chapters which do provide practical details do so in sufficient detail for the book to be a useful manual. To whom, then, will it appeal, because, make no mistake, this is a good and worthwhile book? I find this a very difficult question to answer. It is not, because of the delay between writing and publication a useful introduction to the field, yet it is the sort of book that departments of genetics and cell biology ought to have on their shelves.

\section{JOHN GOSDEN}

Therapy for Genetic Disease. Ed Theodore Friedmann. (Pp 127; $£ 16 \cdot 50$.) Oxford: Oxford University Press. 1991. ISBN 0-19261971-3 (pbk).

Though prenatal diagnosis has provided a valuable tool in helping families with genetic disease, most recognise that this can only ever be a partial solution. Selective abortion will never be entirely acceptable to everyone, and unless all fetuses are screened, affected children will still continue to be born with serious genetic disorders. Many have recognised this dilemma and have hoped that one day effective treatments will be found. This little book addresses this problem. 